Original article

\title{
One Injection of DsRed Followed by Bites from Transgenic Mosquitoes Producing DsRed in the Saliva Elicits a High Titer of Antibody in Mice
}

\author{
Hiroyuki Matsuoka ${ }^{1 *}$, Gen-ichiro Sano ${ }^{1}$, Ryuta Hattori ${ }^{1}$, Hiroyuki Tomita ${ }^{1}$, Daisuke S. Yamamoto ${ }^{1}$ and Makoto Hirai ${ }^{2}$ \\ Received 14 December, 2011 Accepted 23 April, 2012 Published online 4 August, 2012
}

\begin{abstract}
It has been proposed that transgenic mosquitoes can be used as a "flying syringe" for infectious disease control. We succeeded in generating a transgenic (TG) mosquito, Anopheles stephensi, excreting and discharging DsRed in saliva. DsRed was deposited on the membrane where the TG mosquito probed with its proboscis. Repeated feeding by the TG mosquitoes induced anti-DeRed as well as anti-SG antibodies in mice. This indicates that the TG mosquitoes can immunize the animal. Moreover, in this report, we employed a pre-immunization method before exposing mice to the TG mosquitoes. We injected DsRed to mice to prepare memory B cells and exposed the mice to bites by the TG mosquitoes excreting DsRed. The mice produced a higher titer of antibody to DsRed, suggesting that the bites from TG mosquitoes act as a booster and that primary immunization with a vaccine protein and exposure to TG mosquitoes excreting the vaccine protein in the saliva produces a synergistic effect.
\end{abstract}

Key words: Anopheles stephensi, DsRed, flying syringe, salivary gland, transgenic mosquito

\section{INTRODUCTION}

Transgenic techniques have been applied in recent years not only to bacteria but also to animals. Transgenic mice, sheep, dogs, zebra fish, flies and silkworms have appeared in laboratories. Transgenic mosquitoes have also appeared. Anopheline mosquitoes, which transmit malaria parasites, were targeted for the insertion of transgenes to reduce their ability to spread disease [1-3]. Some laboratories, including ours, have succeeded in producing transgenic mosquitoes with a lower level of malaria parasites in the digestive tract after blood meals on malaria-infected animals $[4,5]$. The goal of these endeavors is to control disease transmission through genetic modification of the mosquitoes [6].

We next considered producing a useful protein in the mosquito SG by adding a new gene to a mosquito chromosome, because mosquitoes secrete saliva into the host while taking a bloodmeal. Our idea is to insert a gene encoding a useful protein into the mosquito chromosome, causing the mosquito to produce the protein in its saliva and inject it, via the saliva, into animals or humans upon blood feeding. The host can be expected to develop antibodies to the recombinant protein as a reaction. If TG mosquitoes with saliva containing a vaccine protein against a disease are spread in an area where the disease is prevalent, it follows that people who are bitten daily by the TG mosquitoes will develop antibodies to the vaccine protein, ultimately vaccinating the community against the disease. In this situation, mosquitoes would play the role of vaccine deliverers [7].

We previously discovered a platelet inhibitor in the salivary gland (SG) of the mosquito Anopheles stephensi, cloned its gene, and named the molecule "anopheline antiplatelet protein" (AAPP) [8]. AAPP is expressed only in the female SG. We used an upstream region of the AAPP gene (aapp) as a promoter for a vaccine protein. We have already produced three TG strains of mosquitoes expressing an alien protein in the SG [9-11]. One strain produced a candidate malarial vaccine protein, circumsporozoite protein (CSP), in the SG. However, mice bitten by these mosquitoes did not develop anti-CSP antibodies, because CSP was not discharged outside the SG [9]. The second strain produced SP15, a leishmanial protein, which was discharged into the saliva. Anti-SP15 antibody production was induced in mice

\footnotetext{
${ }^{1}$ Division of Medical Zoology, Department of Infection and Immunity, Jichi Medical University, 3311-1 Yakushiji, Shimotsuke, Tochigi 3290498, Japan

${ }^{2}$ Department of Parasitology, Gunma University Graduate School of Medicine, 3-39-22 Shyowa-machi, Maebashi, Gunma 371-8511, Japan

*Corresponding author:

Tel: +81-285-58-7339

Fax: +81-285-44-6489

E-mail: hiroyuki@jichi.ac.jp
} 
bitten 20 times with 50 of these mosquitoes. It took 20 weeks to detect the antibody [10]. Recently we succeeded in producing a transgenic mosquito expressing a part of CSP in the saliva. The mice bitten by the TG mosquitoes produced anti-CSP antibody, but 20 feedings by 100 TG mosquitoes [11].

We need a more efficient TG mosquito and a more suitable way to elicit the development of antibodies. In the present study, we employed pre-immunization methods before exposing mice to the TG mosquitoes. We injected an immunogen to prepare memory B cells in the mice, and exposed the mice to bites by TG mosquitoes in order to induce a high titer of antibody.

\section{MATERIALS AND METHODS}

\section{Ethics statement}

All care and handling of the animals was conducted in accordance with the Guidelines for Animal Care and Use prepared by Jichi Medical University, following approval (ID: 09-192) by the Jichi Medical University Ethical Review Board.

\section{Mosquitoes and mice}

Adult mosquitoes, Anopheles stephensi SDA 500, were reared in $30 \times 30 \times 30 \mathrm{~cm}$ cages with stainless steel frames and a white cloth covering. The rearing conditions were: $26^{\circ} \mathrm{C}, 50-70 \%$ relative humidity, 13 hours of light and 11 hours of darkness. Adult females could draw blood through the cloth when a mouse was anesthetized and laid on the top of the cage. Female ICR mice were purchased from SLC (Shizuoka, Japan).

\section{DsRed protein, antibody and DNA}

A recombinant DsRed-monomer protein and a rabbit antibody to DsRed-monomer were purchased from Clontech Laboratories (Mountain View, CA, USA). We also purchased DsRed monomer vector (Clontech Laboratories) for the preparation of template DNA.

\section{Plasmid construction (Fig. 1)}

A DNA fragment (1,746 bp) covering the anopheline anti-platelet protein gene (aapp) promoter region and signal peptide coding region of An. stephensi (GeneBank accession no. AB212871.1) was amplified with the primers AAPP-attB4F (5'-GGGGACAACTTTGTATAGAAAAGTTG TTATAAGACGGAGCTCATTGTCGCTCGTC-3') and AAPP-attB1R (5'-GGGGACTGCTTTTTTGTACAAACTTG CGGCCGTGCGGATACGATCAGCGCAAGGC-3'), then cloned into the vector pDONR ${ }^{\mathrm{TM}} \mathrm{P} 4-\mathrm{P} 1 \mathrm{R}$ (Invitrogen, Carlsbad, CA, USA) (plasmid a). The dsRed monomer gene (Invitrogen)

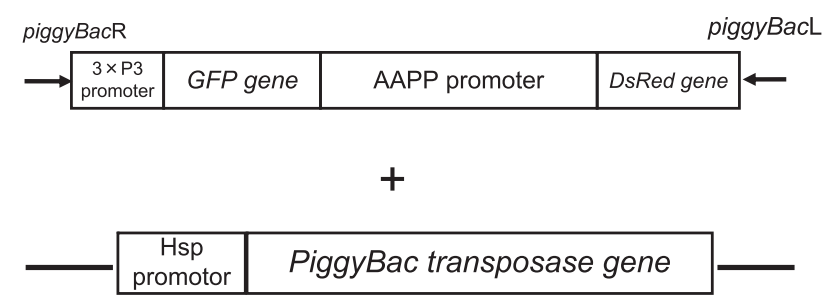

Fig. 1. DNA injected into mosquito eggs. Two plasmids were injected. One contained GFP and DsRed genes with piggyBac arms. The other contained the PiggyBac transposase gene. 3xP3: a protein expressed in the eyes (fragment size $41 \mathrm{bp}$ ). GFP: Green fluorescent protein as a marker of eyes (717 bp). AAPP: Anopheline antiplatelet protein (1746 bp). DsRed: a marker of saliva from the salivary gland expected to be secreted in the saliva (675 bp). Hsp: Heat shock protein (393 bp).

was amplified with dsRed-attB1F (5'-GGGGACAAGTTTG TACAAAAAAGCAGGCTATGAAGCTTGCCTCCTCCGA GAACGTC-3') and dsRed-attB2R (5'-GGGGACCACTTTG TACAAGAAAGCTGGGTTTAGTGGTGGTGGTGGTGGTG-3'), then cloned into the vector pDONR ${ }^{\mathrm{TM}} 221$ (plasmid b). The 3 ' non-coding region of the AAPP gene was amplified with 3UTR-attB2F (5'-GGGGACAGCTTTCTTGTACAAAGTGG GAAACACACCGTTAACGACAC-3') and 3UTR-attB3R (5'-GGGGACAACTTTGTATAATAAAGTTGTATTCAAAG GTCCACAAATGTC-3'), and cloned into the vector pDONRP2R-P3 ${ }^{\mathrm{TM}}$ (plasmid c). The pBac [3xP3-EGFP] vector and helper plasmid were kindly provided by Professor A. S. Raikhel (Department of Entomology, University of California at Riverside, USA) [12]. The pBac [3xP3EGFP] vector was digested with AscI, blunted with a Klenow fragment, and dephosphorylated. The reading frame cassette A (Invitrogen) was then cloned into the vector (destination vector). The inserts of plasmids $\mathrm{a}, \mathrm{b}$ and $\mathrm{c}$ were serially ligated in destination vector using the MultiSite Gateway $^{\circledR}$ Three Fragment Vector Construction Kit (Invitrogen).

The resulting plasmid (pBac-AAPP-DsRed) was expected to drive expression of the dsRed gene under the control of the AAPP promoter. The pBac-AAPP-dsRed plasmid was mixed with piggyBac helper (Fig. 1) and microinjected into the eggs of $A$. stephensi.

\section{Microinjection of the plasmids into mosquito eggs}

The microinjection was performed as described elsewhere [13]. In brief, blood-fed An. stephensi mosquitoes were allowed to oviposit on a wet filter sheet 72-84 h after a blood meal. Eggs were injected within 120 minutes of oviposition. The injection was done using glass needles (Eppendorf, Hamburg, Germany) with a mixture of pBac- 
AAPP-DsRed (400 ng/ $\mu \mathrm{l})$ and piggyBac helper (100 ng/ $\mu \mathrm{l})$ in injection buffer ( $5 \mathrm{mM} \mathrm{KCl,} 0.1 \mathrm{mM} \mathrm{Na}_{2} \mathrm{HPO}_{4}, \mathrm{pH}$ 6.8). The eggs were then placed in water and observed for hatching. The hatched larvae were analyzed on a fluorescence microscope at a wavelength of $488 \mathrm{~nm}$ to detect GFP expression.

\section{Selection of transgenic mosquitoes}

We reared the hatched larvae and allowed them to emerge as adults. One adult mosquito was placed in a cage containing five wild-type adult mosquitoes of the opposite sex. After mating and blood feeding, each female was allowed to lay eggs individually. The hatched larvae were observed under a fluorescence microscope, and GFPexpressing larvae were isolated as a G1 strain. Among the same G1 batch, mosquitoes were allowed to mate, feed, and lay eggs. The hatched larvae were observed, and the GFPexpressing larvae were isolated as a G2 strain.

\section{Western blotting}

Three to four days after emergence, ten pairs of SG were collected from both TG females and wild-type females in ten micro-liters of phosphate-buffered saline (PBS) in an Eppendorf tube. The tube was frozen at $-80^{\circ} \mathrm{C}$ and thawed at room temperature. It was then centrifuged at 8,000 rpm for $3 \mathrm{~min}$. The supernatant was used as the SG antigen assuming that one pair of SG protein was contained in one micro-liter. Half of the samples (five pairs of SG) were separated on a $10 \%$ SDS-polyacrylamide gel under reducing conditions (with 2\% 2-mercaptoethanol) and transferred to a nitrocellulose (NC) sheet. The sheet was soaked in $1 \%$ skim milk for ten minutes, and probed with a rabbit antiDsRed antibody (Clontech) diluted 1,000 fold. The sheet was washed with $0.05 \%$ Tween 20 in PBS four times and then incubated with anti-rabbit IgG conjugated with HRP (Bio-Rad Lab.) diluted 3,000 fold. The sheet was washed with $0.05 \%$ Tween 20 in PBS four times and reacted with Super Signal West Pico Chemiluminescent Substrate (Thermo Fisher Scientific Inc., Rockford, IL, USA). Positive bands were visualized with a Lumino Image Analyzer (LAS-1000) (Fuji Film, Tokyo, Japan).

\section{Observation of the SG}

An adult female mosquito was dissected three days after emergence. A pair of SG was isolated and examined under a confocal microscope, FV1000 (Olympus Co., Tokyo, Japan) to observe the red color of DsRed in the SG.

\section{Discharge of recombinant DsRed from TG mosquitoes}

To observe the discharge of DsRed from the proboscis of the TG mosquitoes, we employed the method of
Billingsley et al. [14] with some modifications. We put a $30 \times 40 \mathrm{~mm}$ piece of NC sheet on top of the mosquito cage and placed a $30-\mathrm{ml}$ trianglular flask containing $40-45^{\circ} \mathrm{C}$ of water on the NC sheet. The mosquitoes sensed the higher temperature at the top of the cage, gathered at the location on the sheet, and started probing for blood capillaries. In 10 minutes, they deposited saliva on the NC sheet. We cut the sheet into four pieces and incubated each with the rabbit anti-DsRed antibody (Clontech), normal rabbit serum, the mouse anti-SG antibody [9] and normal mouse serum. These sheets were reacted with HRP-conjugated anti-rabbit IgG or anti-mouse IgG, then probed with substrate solution. Positive spots were visualized with the LAS-1000.

\section{TG mosquito biting on naïve mice}

To produce anti-DsRed antibodies, two naïve ICR mice (No. 1 and No. 2) were each bitten by 50 female DsRed-TG mosquitoes every two weeks for ten weeks (five times). Anti-DsRed antibody production was monitored by ELISA as shown below. As a negative control, two ICR mice (No. 3 and No. 4) were each bitten by 50 female wildtype mosquitoes every two weeks for ten weeks. Anti-SG antibody production was monitored by ELISA.

\section{Pre-immunization of mice with DsRed}

Six ICR mice were peritoneally injected with $1 \mu \mathrm{g}$ of DsRed plus Alum. Two weeks later, two of the mice (No. 5 and No. 6) were bitten by 50 DsRed-TG mosquitoes. The biting was repeated five times at two-week intervals. Two other mice (No. 7 and No. 8) were each bitten by 50 wildtype mosquitoes at two-week intervals. Two more mice (No. 9 and No. 10) were injected with $1 \mu$ g of DsRed plus Alum one more time but not bitten by mosquitoes.

\section{Evaluation of antibody concentrations by ELISA}

For the evaluation of anti-DsRed antibody production, 30 wells of a 96-well assay plate were coated with $100 \mu \mathrm{l}$ of DsRed $(1 \mu \mathrm{g} / \mathrm{ml})$ (Clontech) in $0.05 \mathrm{M}$ carbonate buffer ( $\mathrm{pH}$ 9.6). To evaluate anti-SG antibody production, 40 pairs of SG were collected from wild-type female mosquitoes, disrupted in $4.0 \mathrm{ml}$ of carbonate buffer by sonication ( $1 \mathrm{sec} \times 5$ times), and centrifuged at 8,000 rpm for $3 \mathrm{~min}$. The supernatant was used as SG antigen. Thirty wells were coated with $100 \mu \mathrm{l}$ of SG antigen. Another 30 wells were filled with $100 \mu$ l of carbonate buffer without antigen. The plate was incubated at $4^{\circ} \mathrm{C}$ overnight.

Five micro-liters of blood was collected from the tail of each mouse 7 days after the final mosquito biting. The blood was mixed with $1.0 \mathrm{ml}$ of phosphate-buffered saline containing $1 \%$ bovine serum albumin (BSA/PBS), and centrifuged at 8,000 rpm for $3 \mathrm{~min}$. The supernatant was used 
as 400-fold diluted serum.

After removal of the antigen solution and two washes, the wells of the ELISA plate were blocked with $150 \mu \mathrm{l}$ of $1 \%$ BSA/PBS for $30 \mathrm{~min}$. The blocking solution was removed, and $100 \mu \mathrm{l}$ of mouse serum diluted 400-fold was added to three DsRed antigen-containing wells, three SG antigen-containing wells and three antigen-free wells. After $2 \mathrm{~h}$ of incubation and five washes, a secondary antibody, rabbit anti-mouse IgG conjugated with horseradish peroxidase (HRP) (Bio-Rad) diluted 3,000-fold, was added. After $1 \mathrm{~h}$ of incubation and five washes, a substrate solution, a mixture of $0.04 \%$ 2,2'-azino-bis(3-ethylbenzthiazoline-6sulfonic acid) (ABTS) (Sigma-Aldrich, St. Louis, MO, USA), $0.05 \% \mathrm{H}_{2} \mathrm{O}_{2}$ in $0.05 \mathrm{M}$ phosphate and $0.1 \mathrm{M}$ citrate buffer ( $\mathrm{pH} 4.5$ ), was added. After 20-60 min, a green color appeared. Absorption was measured at a wavelength of 405 $\mathrm{nm}$. The mean absorbance of antigen-containing wells minus that of antigen-free wells was used as the OD value.

\section{RESULTS}

\section{Isolation of TG mosquitoes}

Two recombinant plasmids (Fig. 1) were injected into 117 eggs of wild-type mosquitoes. Forty-four larvae hatched and 38 of these grew and emerged. Adult females were mated with wild-type males, and adult males were mated with wild-type females, and then eggs were obtained from each female individually. After hatching, the GFPexpressing larval group was considered to be a transgenic mosquito strain. In the end, four strains were established.

\section{Molecular size of recombinant DsRed produced in the SG}

Three to five days after emergence, ten pairs of SG were collected from female TG mosquitoes in each colony. Half of the samples were separated on a SDSpolyacrylamide (10\%) gel under reducing conditions and transferred to a NC sheet. Western blotting produced bands corresponding to a molecular size of $28 \mathrm{kDa}$. Among the four colonies, \#4 expressed DsRed most strongly (data not shown). A comparison of the density of the $28 \mathrm{kDa}$ band with that of the positive control bands for a series of different amounts of DsRed monomer revealed that the amount of DsRed in the \#4 lanes (5 and 10 pairs, respectively) was equivalent (in reactivity with the anti-DsRed antibody) to 125 and 250 ng of DsRed monomer (Fig. 2). This, in turn, indicated that approximately $25 \mathrm{ng}$ of DsRed was expressed in one pair of SG in the \#4 TG mosquitoes. We chose colony \#4 for the subsequent experiments.

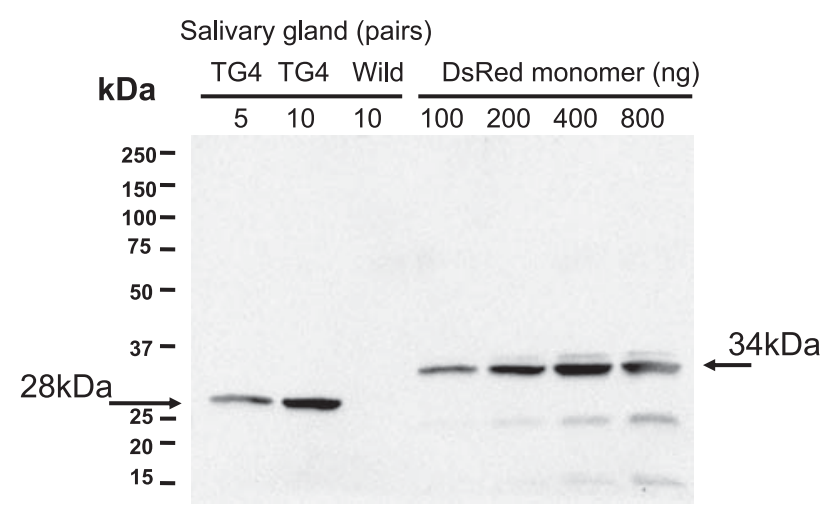

Fig. 2. Estimation of DsRed amount produced in the SG of \#4 DsRed-TG mosquitoes. Five and ten pairs of SG of DsRed-TG mosquitoes were separated, and compared with a series of different amounts of DsRed monomer (34 kDa).

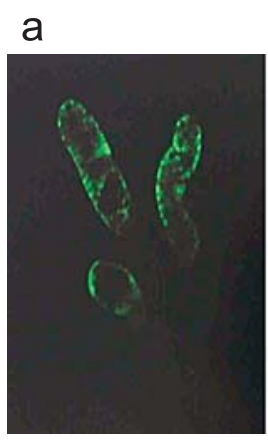

b
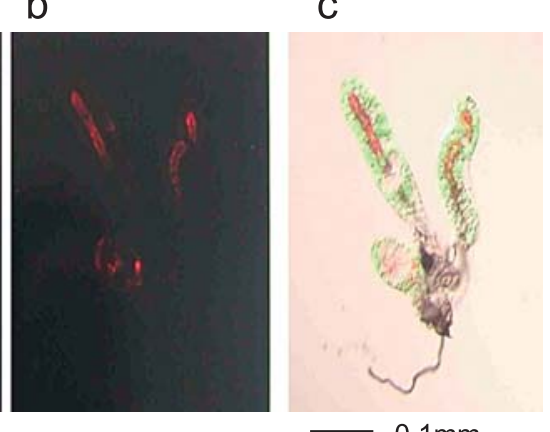

$0.1 \mathrm{~mm}$
Fig. 3. Salivary gland of TG mosquitoes expressing GFP and DsRed. a: GFP expressed in salivary gland cells. b: DsRed in the lumen of the salivary gland. c: combined photograph.

\section{Observation of the SG}

An adult female TG mosquito was dissected three days after emergence, and the SG was isolated and observed under a confocal microscope. As shown in Fig. 3, the green color of GFP was observed in cellular areas where saliva proteins were produced. The red color of DsRed was observed in the lumen, the cavity for storing saliva, in the central part of the SG. This indicates that an alien protein, DsRed, was secreted into the cavity of the SG.

\section{Discharge of recombinant DsRed from the SG}

As shown in Fig. 4, mosquito saliva was deposited on the NC sheets. Spots of DsRed were detected on the sheet probed by the DsRed-TG mosquitoes. This is direct evidence that an alien protein, DsRed, was discharged on the NC sheet from the TG mosquitoes. 


\section{Development of anti-DsRed antibody through TG mos- quito bites}

After being bitten repeatedly by the DsRed-TG mosquitoes, ICR mice developed anti-DsRed as well as anti-SG antibodies in their serum (Fig. 5). This was indirect evidence that the TG mosquitoes deposited DsRed together

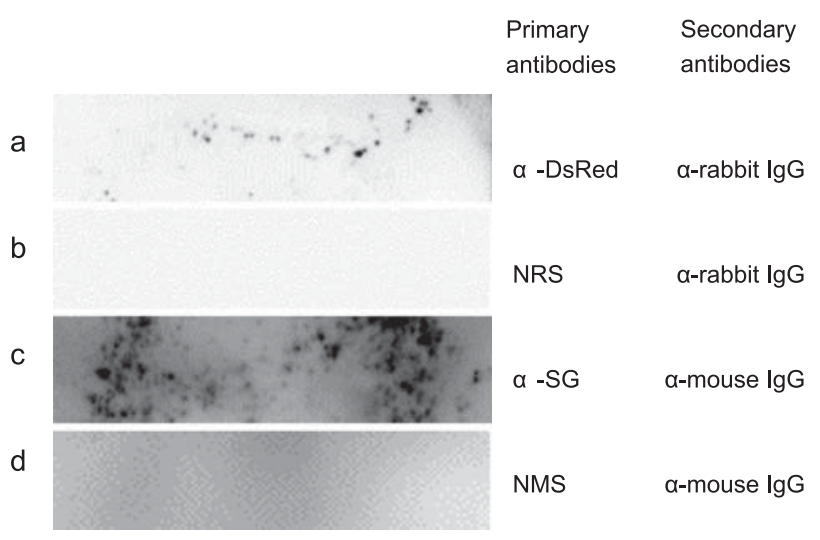

Fig. 4. Discharge of recombinant DsRed from TG mosquitoes. Spots on NC sheets are saliva deposited by DsRed-TG mosquitoes. NRS: normal rabbit serum. NMS: normal mouse serum.

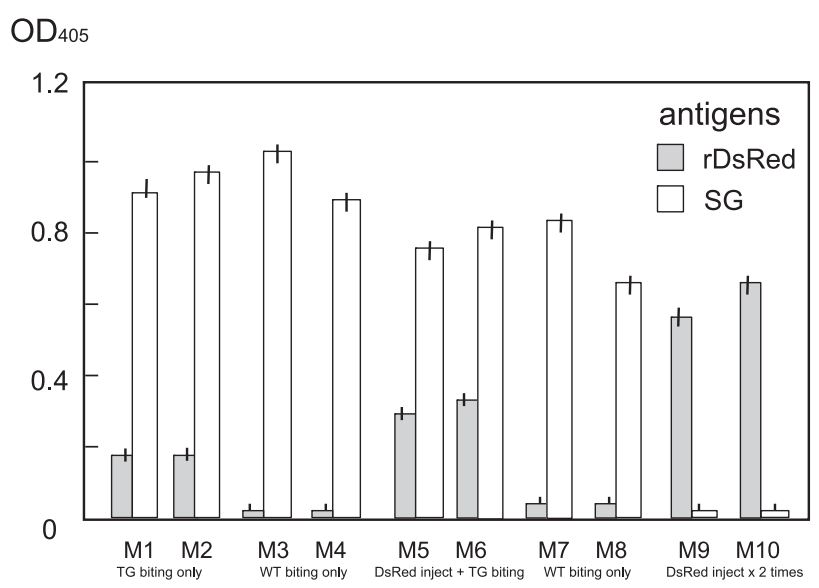

Fig. 5. Development of anti-DsRed antibody by DsRed-TG mosquito bites. Mice No. 1 and No. 2 were each bitten by 50 DsRed-TG mosquitoes five times at two-week intervals. Anti-DsRed antibody was produced in the two mice. Mice No. 3 and No. 4 were each bitten by 50 wild-type mosquitoes five times. Mice No. 5-10 were injected with $1 \mu \mathrm{g}$ of DsRed. Then mice No. 5 and No. 6 were each bitten by 50 DsRed-TG mosquitoes five times at two-week intervals. Much anti-DsRed antibody was produced in the two mice. Mice No. 7 and No. 8 were each bitten by 50 wild-type mosquitoes five times. Mice No. 9 and No. 10 were injected with $1 \mu \mathrm{g}$ of DsRed one more time. with saliva into the mouse skin when they drew blood. In the next experiment, we injected $1 \mu \mathrm{g}$ of DsRed monomer into ICR mice with Alum before any mosquito biting. The pre-immunized mice (No. 5 and 6) produced anti-DsRed antibody after repeated biting by the TG mosquitoes. Compared with mice No. 1 and No. 2, mice No. 5 and No. 6 produced more anti-DsRed antibody. However, No. 9 and No. 10 , which were injected with DsRed two times, produced more anti-DsRed antibody than No. 5 and No. 6.

\section{DISCUSSION}

Mosquitoes deposit saliva in the skin when they take a bloodmeal, causing a variety of physiological responses in the host [15]. Repeated biting leads to the development of an anti-saliva antibody [16]. In areas where malaria is hyper-endemic, more than 100 anopheline mosquitoes can attack a person in a single hour during the night [17]. In these areas, therefore, most people have high levels of antisaliva antibodies [18-20]. It follows that if transgenic (TG) mosquitoes expressing a vaccine protein in saliva could be produced and released by the millions into the field, people would be supplied with the protein when they were bitten, produce antibodies to the protein, and develop immunity to the disease.

In 2010, we succeeded in producing a TG mosquito expressing GFP in the eyes and producing a recombinant CSP in the SG [9]. We allowed a hundred of the CSPexpressing TG mosquitoes (CSP-TG mosquitoes) to feed on mice several times, but the mice only developed anti-saliva antibodies. The reason for this might be that the TG mosquito produced CSP in the salivary gland cells but did not excrete it in the saliva and so did not inject it into the mice. In this study, we employed a colored protein, DsRed, in order to observe the location of the alien protein under a fluorescence microscope. As expected, we observed that the TG mosquitoes excreted DsRed in the cavity of the SG (Fig. 3). Moreover, the TG mosquitoes discharged DsRed via their proboscis (Fig. 4).

The estimated amount of DsRed in the salivary grand was 25 ng per individual female mosquito (Fig. 2). In our previous experiments, the estimated amount of transgenic protein in the salivary gland was $40 \mathrm{ng}$ of CSP [9], $20 \mathrm{ng}$ of SP15 [10], and $10 \mathrm{ng}$ of DsRed-CSP [11]. These amounts were not significantly different.

The mice bitten by DsRed-TG mosquitoes produced anti-DsRed antibodies as expected (Fig. 5), but the titer was not as high as that of the anti-SG antibody. In the next experiment, we injected $1 \mu \mathrm{g}$ of DsRed intraperitoneally once and allowed the mice to be fed upon by DsRed-TG mosquitoes. After several rounds of biting, the mice developed a 
higher concentration of anti-DsRed antibody than did the mice only bitten by DsRed-TG mosquitoes (Fig. 5). Memory B cells might be produced in the mice in response to the first injection of DsRed. The DsRed-TG mosquitoes' biting might then act as a booster. This suggests that the primary immunization of babies with a malarial vaccine protein and then exposure to TG mosquitoes excreting the malarial vaccine protein in saliva would produce a synergistic effect in endemic areas.

In our previous report, we allowed mice to be bitten 20 times by 100 TG mosquitoes. Over four months and about 1,500 bites were needed for the suitable antibody titer to inhibit the invasion of malaria parasites to hepatic cells [11]. However, pre-immunizing methods may reduce the number of mosquito bites. With one pre-immunization in a mouse, biting by 50 TG mosquitoes five times should be enough to induce a similar titer of anti-malarial antibody in the mouse. Further experiments are needed to confirm this possibility.

\section{ACKNOWLEDGEMENTS}

We thank Ms. Chisato Seki and Ms. Michiyo Soutome for assistance with the ELISA and Western blotting, and Ms. Keiko Watano and Mr. Jin Sato for handling of the mosquitoes and mice. This work was supported by a grant from the Bill \& Melinda Gates Foundation through the Grand Challenges Exploration Initiative (Grant No. 51694) to HM, and a grant of the Support Program for Strategic Research Platform for Private Universities from the Japanese Ministry of Education, Culture, Sport, Science and Technology to HM.

\section{CONFLICT OF INTEREST}

The authors have no conflict of interest.

\section{REFERENCES}

1. Alphey L, Beard CB, Billingsley P, Coetzee M, Crisanti A, Curtis C, Eggleston P, Godfray C, Hemingway J, JacobsLorena M, James AA, Kafatons FC, Mukwaya LG, Paton M, Powell JR, Schneider W, Scott TW, Sina B, Sinden R, Sinkins S, Spielman A, Touré Y, Collins FH. Malaria control with genetically manipulated insect vectors. Science 2002; 298: 119-121.

2. Nirmala X, James AA. Engineering Plasmodium-refractory phenotypes in mosquitoes. Trends Parasitol 2003; 19: 384-387.

3. Riehle MA, Srinivasan P, Moreira CK, Jacobs-Lorena M. Towards genetic manipulation of wild mosquito populations to combat malaria: advances and challenges. J Exp Biol 2003; 206: 3809-3816.
4. Ito J, Ghosh A, Moreira LA, Wimmer EA, Jacobs-Lorena M. Transgenic anopheline mosquitoes impaired in transmission of a malaria parasite. Nature 2002; 417: 452-455.

5. Yoshida S, Shimada Y, Kondoh D, Kouzuma Y, Ghosh AK, Jacobs-Lorena M, Sinden RE. Hemolytic C-type lectin CEL-III from sea cucumber expressed in transgenic mosquitoes impairs malaria parasite development. PLoS Pathog 2007; 3: e192.

6. Crampton JM, Warren A, Lycett GJ, Hughes MA, Comley IP, Eggleston P. Genetic manipulation of insect vectors as a strategy for the control of vector-borne disease. Ann Trop Med Parasitol 1994; 88: 3-12.

7. Crampton JM, Stowell SL, Karras M, Sinden RE. Model systems to evaluate the use of transgenic haematophagous insects to deliver protective vaccines. Parassitologia 1999; 41: 473-477.

8. Yoshida S, Sudo T, Niimi M, Tao L, Sun B, Kambayashi J, Watanabe H, Luo E, Matsuoka H. Inhibition of collageninduced platelet aggregation by anopheline antiplatelet protein, a saliva protein from a malaria vector mosquito. Blood 2008; 111: 2007-2014.

9. Matsuoka H, Ikezawa T, Hirai M. Production of a transgenic mosquito expressing circumsporozoite protein, a malarial protein, in the salivary gland of Anopheles stephensi (Diptera: Culicidae). Acta Med Okayama 2010; 64: 233241.

10. Yamamoto DS, Nagumo H, Yoshida S. Flying vaccinator; a transgenic mosquito delivers a Leishmania vaccine via blood feeding. Insect Mol Biol 2010; 19: 391-398.

11. Yamamoto DS, Sumitani M, Nagumo H, Yoshida S, Matsuoka $\mathrm{H}$. Induction of anti-sporozoite antibodies by biting of transgenic Anopheles stephensi delivering malarial antigen via blood feeding. Insect Mol Biol 2012; 21: 223-233.

12. Cho KH, Cheon HM, Kokoza V, Raikhel AS. Regulatory region of the vitellogenin receptor gene sufficient for highlevel, germ line cell-specific ovarian expression in transgenic Aedes aegypti mosquitoes. Insect Biochem Mol Biol 2006; 36: 273-281.

13. Catteruccia F, Nolan T, Loukeris TG, Blass C, Savakis C, Kafatos FC, Crisanti A. Stable germline transformation of the malaria mosquito Anopheles stephensi. Nature 2000; 405: 959-962.

14. Billingsley PF, Hodivala KJ, Winger LA, Sinden RE. Detection of mature malaria infections in live mosquitoes. Trans Roy Soc Trop Med Hyg 1991; 85: 450-453.

15. Clements AN. The adult salivary glands and their secretions. In: The Biology of Mosquitoes. London: Chapman \& Hall; 1992. pp. 251-262.

16. Penneys NS, Nayar JK, Bernstein H, Knight JW, Leonardi C. Mosquito salivary gland antigens identified by circulating human antibodies. Arch Dermatol 1989; 125: 219-222.

17. Harada M, Ikeshoji T, Suguri S. Studies on vector control by "Mosquito Candle”. In: Ishii A, Nihei N, Sasa M, eds. Malaria Research in the Solomon Islands. Tokyo: Inter Group Corporation; 1998. pp. 120-125.

18. Waitayakul A, Somsri S, Sattabongkot J, Looareesuwan S, 
Cui L, Udomsangpetch R. Natural human humoral response to salivary gland proteins of Anopheles mosquitoes in Thailand. Acta Trop 2006; 98: 66-73.

19. Andrade BB, Rocha BC, Reis-Filho A, Camargo LM, Tadei WP, Moreira LA, Barral A, Barral-Netto M. AntiAnopheles darlingi saliva antibodies as marker of Plasmodium vivax infection and clinical immunity in the Brazilian
Amazon. Malar J 2009; 8: 121.

20. Rizzo C, Ronca R, Fiorentino G, Verra F, Mangano V, Poinsignon A, Sirima SB, Nèbiè I, Lombardo F, Remoue F, Coluzzi M, Petrarca V, Modiano D, Arcà B. Humoral response to the Anopheles gambiae salivary protein gSG6: a serological indicator of exposure to Afrotropical malaria vectors. PLoS One 2011; 6: e17980. 